\title{
WHAT IS NEW IN ASTROPARTICLE PHYSICS
}

\author{
Franco Giovannelli*
}

\author{
INAF - Istituto di Astrofisica e Planetologia Spaziali, Area di Ricerca di Tor Vergata, Via del Fosso del \\ Cavaliere, 100 - I00177 Roma, Italy \\ * corresponding author: franco.giovannelli@iaps.inaf.it
}

ABstRaCT. In this brief review paper I will point to the most important steps that have been made in recent decades toward a better understanding of the physics governing our Universe. Because of the limited length of this paper, I have selected only a few results that, in my opinion, have been of crucial importance.

KEYWORDS: photonic astrophysics, particle astrophysics, neutrino astrophysics.

\section{INTRODUCTION}

Astroparticle Physics is a new field of physics that emerged roughly twenty years ago, when high energy astrophysicists and particle physicists began to collaborate. Within this relatively short period of time, astroparticle physics has developed strongly through the study of cosmic sources that are emitters of photons, charged particles and neutrinos. These sources are considered as frontier objects between astrophysics and particle physics. Results coming from the study of cosmic sources using various techniques have stimulated the scientific community to work toward a unifying scheme for a general comprehension of the physics governing our Universe.

Wilkinson Microwave Anisotropy Probe (WMAP) Mission described by Bennett et al. [25] determined that the universe is $13.7 \pm 0.2 \mathrm{Gyr}$ old. The combination of WMAP and 2dFGRS data favors a flat universe, from which it follows that the mean energy density in the universe is equal to the critical density [158. This is equivalent to a mass density of $9.9 \times 10^{-30} \mathrm{~g} \mathrm{~cm}^{-3}$. Of this total density, we now know the breakdown to be $4.6 \%$ baryons, $0.4 \%$ neutrinos, $23 \%$ Cold Dark Matter (CDM), and $72 \%$ Dark Energy [81] and references therein).

Till now, we have no unquestionable experimental proof of the existence of dark matter and dark energy, though some results on the presence of DM particles in the galactic halo have been claimed by Bernabei et al. [26]. Bernabei et al. [27] (this volume) point out that the DAMA/LIBRA data shows a modelindependent evidence of the presence of DM particles in the galactic halo at $8.9 \sigma$ C.L. If this is so, it will greatly improve our knowledge of the universe.

However, Ackerman et al. [7] observing the satellite galaxies of the Milky Way - the most promising targets for dark matter searches in gamma rays - did not find any positive signal. In their search for dark matter consisting of weakly interacting massive particles - applying a joint likelihood analysis to 10 satellite galaxies with 24 months of data from the Fermi Large
Area Telescope - no dark matter signal was detected. Including the uncertainty in the dark matter distribution, robust upper limits are placed on dark matter annihilation cross sections. The $95 \%$ confidence level upper limits range from about $10^{-26} \mathrm{~cm}^{3} \mathrm{~s}^{-1}$ at $5 \mathrm{GeV}$ to about $5 \times 10^{-23} \mathrm{~cm}^{3} \mathrm{~s}^{-1}$ at $1 \mathrm{TeV}$, depending on the dark matter annihilation final state.

All cosmic sources, both discrete and diffuse, are variable in intensity and in spectral shape at different time scales. In this sense, we can affirm that no single source is sufficiently stable to be considered a standard candle. For this reason, multifrequency observations, possibly simultaneous, are mandatory for a proper comprehension of the behaviour of a target cosmic source (e.g. [81]).

In this paper I will discuss - following my knowledge and feelings - the most relevant results obtained in the recent past that significantly improved our knowledge of the physics governing our universe. Deeper discussions about astroparticle physics can be found in review papers by Giovannelli [79, 81]. In their review papers, Giovannelli \& Sabau-Graziati [92, 94, and De Angelis, Mansutti \& Persic [53] have discussed the multi-frequency behaviour of high energy cosmic sources, and very high energy (VHE) $\gamma$-ray astrophysics. The results from EGRET have been extensively discussed by Thompson [170] in his review of $\gamma$-ray astrophysics.

\section{THE MAIN PILLARS OF ASTROPARTICLE PHYSICS}

High Energy Astrophysics is generally approached through the study of cosmic rays. The reason for this is historical in nature. Since the discovery of this extraterrestrial radiation by Victor Hess [103, an enormous amount of scientific research has been involved in trying to discover its nature, and as a result many separate research fields have been developed. Before particle accelerators came into operation high energy cosmic rays were the laboratory tools for investigations of elementary particle production, 
and to date they are still the only source of particles with energies greater than $10^{12} \mathrm{eV}$. Research into the composition of radiation led to the development of studies of the astrophysical environment using the information in the charge, mass, and energy spectra; this field is also known as Particle Astrophysics.

The discovery of high energy photons near the top of Earth's atmosphere was of great importance, and led to the development of new astronomical fields such as $\mathrm{X}$-ray and $\gamma$-ray astronomy. However, many of these high energy photons have their origin in interactions of high energy charged particles with cosmic matter, light, or magnetic fields. The research fields of particle astrophysics and astronomy have found in this fact a bond to join their efforts in trying to understand the high energy processes which occur in astrophysical systems.

\subsection{COSMIC RAYS}

The modern picture of cosmic rays is that of a steady rain of particles moving at speeds close to that of light. The particles are primarily nuclei with atomic weights less than 56 , as well as a few nuclei of heavier elements, some electrons and positrons, a few $\gamma$-rays and neutrinos.

The energy spectrum extends over 12 orders of magnitude $\left(\sim 10^{8} \div 10^{20} \mathrm{eV}\right)$ and the particle flux rapidly decreases with increasing energy. Figure 1 shows the energy spectrum of cosmic rays. Until energies of $\sim 10^{9} \mathrm{eV}$, CRs are of galactic origin and suffer a strong solar modulation due to their relatively low energy. From $\sim 10^{9} \mathrm{eV}$ to $\sim 10^{15} \mathrm{eV}, \mathrm{CRs}$ are still of galactic origin and probably are mainly accelerated by supernova remnants (SNRs). From $\sim 10^{15} \mathrm{eV}$ to $\sim 10^{19} \mathrm{eV}$, CRs shows some hints of galactic anisotropy at $\sim 10^{18} \mathrm{eV}$, and composition from heavy to light elements. Above $\sim 10^{19} \mathrm{eV}$, the ultra high energy (UHE) CRs are very few in number, and their origin and composition is mostly unknown. Figure 1 shows clearly that the CR flux falls from $1 \mathrm{CR} \mathrm{m}^{-2} \mathrm{~s}^{-1}$ at $\sim 10^{11} \mathrm{eV}$, to $1 \mathrm{CR} \mathrm{m}^{-2} \mathrm{yr}^{-1}$ at $\sim 10^{15 \div 16} \mathrm{eV}$, to $1 \mathrm{CR} \mathrm{km}^{-2} \mathrm{yr}^{-1}$ at $\sim 10^{19} \mathrm{eV}$.

There is evidence of a break in the spectrum around $10^{15} \div 10^{16} \mathrm{eV}$. This break is also called the knee. The knee was observed in the late 1950s initially as a steepening in the extensive air shower (EAS) size spectrum [120]. Since then more than 60 years have passed but its origin is still a challenge for cosmic ray physics.

A possible interpretation of the knee is that it represents the energy at which cosmic rays can escape more freely from the Galaxy, or it may indicate a transition between two different acceleration mechanisms. In the first case, one might expect an anisotropy effect in the distribution of arrival directions above this energy if the cosmic rays originated within the Galaxy.

A $2^{\text {nd }}$ knee is present at about $10^{18} \mathrm{eV}$. Its origin is not yet completely clear: it could be due to dispersion of SNs, or re-acceleration of particles or early

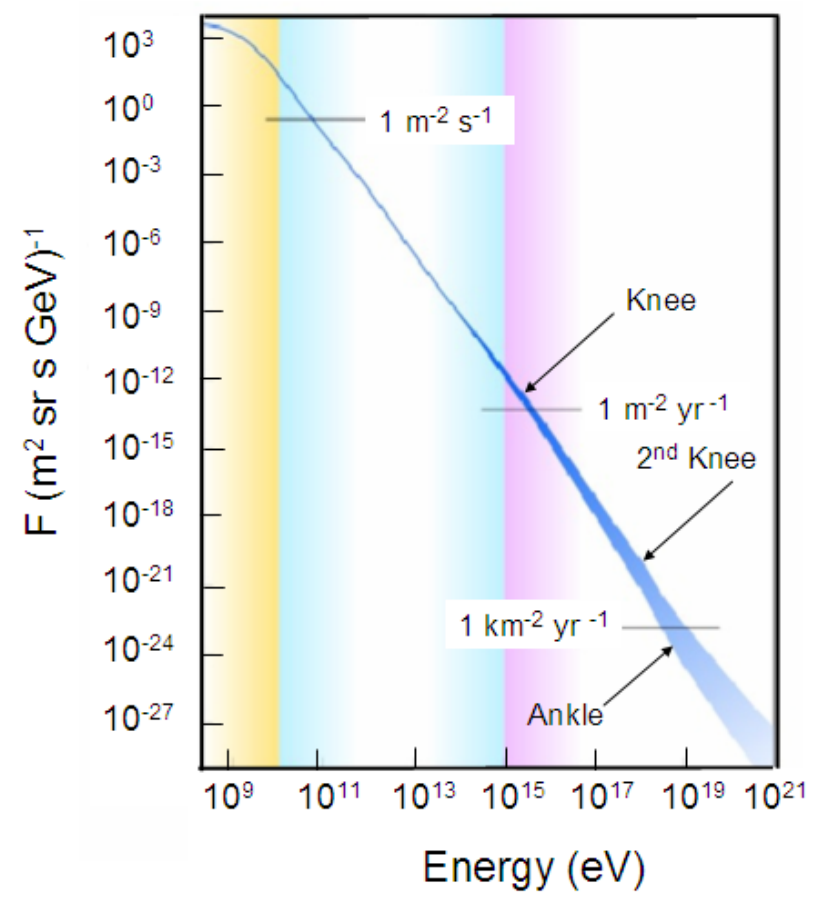

Figure 1. The whole particle spectrum of cosmic rays (after the talk by Mostafa, 2012 132]). Energies less or greater than $\approx 10^{15} \mathrm{eV}$ divide the two ranges in which direct and indirect measurements of the CR spectrum are possible.

transition to extragalactic cosmic rays 134 .

In summary at $\mathrm{GeV}$ energies the cosmic ray spectrum for protons is close to $E^{-2.75}$, and for He and higher elements it is close to $E^{-2.65}$ below the knee at $\approx 5 \times 10^{15} \mathrm{eV}$, where the spectrum turns down to $\sim E^{-3.1}$, to and flattens out again near $10^{18.5} \mathrm{eV}$, called the ankle (e.g., [123, 133, 183]).

The origin of the highest energy CRs remains an open question. For a general review of high energy CRs, see [160].

The Greisen-Zatsepin-Kuzmin (GZK) cutoff is due to the microwave background, near to $10^{21} \mathrm{eV}$. Once we succeed in identifying the origin of the highest energy events beyond $5 \times 10^{19} \mathrm{eV}$, and if we can establish the nature of their propagation through the universe to us, then we will obtain a tool for doing physics at $\mathrm{EeV}$ energies 28.

Dirac's famous statement, from his Nobel Lecture speech, is: We must regard it rather as an accident that the Earth (and presumably the whole Solar System), contains a preponderance of negative electrons and positive protons. It is quite possible that for some of the stars it is the other way about, these stars being built up mainly of positrons and negative protons. This statement still poses a fundamental question - open to experimental observations - about the baryonic symmetry or asymmetry of the Universe. This topic can be investigated either 'indirectly', by measuring the spectrum of the cosmic diffuse gamma-ray, or 'directly', by searching for anti-nuclei and by measuring the energy spectra of antiprotons and positrons. 
The PAMELA satellite, designed to perform accurate measurements of cosmic rays, has revealed antiprotons and positrons in the range $\sim 10 \div 100 \mathrm{GeV}$, providing important constraints for the existence of exotic processes and information about mechanisms for the production, acceleration and propagation of CRs in the Galaxy (e.g. 69]). As annihilating dark matter particles in many models are predicted to contribute to the cosmic ray positron spectrum in this energy range, a great deal of interest has resulted from this observation. However, pulsars could be an alternative source of this signal [106. The excesses recently observed in the cosmic ray positron and electron spectra by the PAMELA and ATIC experiments could be produced by a nearby clump of $600 \div 1000 \mathrm{GeV}$ neutralinos [107]. Shaviv, N., Nakar \& Piran [154] showed that inhomogeneity of CR sources, due to the concentration of supernova remnants (SNRs) towards the galactic spiral arms, can provide a natural explanation for the anomalous increase in the positron/electron ratio observed by PAMELA.

The propagation of charged CRs is influenced by the magnetic field in the Galaxy, and for the lowest energy particles also in the solar system. The result is that the distribution of arrival directions as the radiation enters Earth's atmosphere is nearly isotropic. It is not possible to identify the sources of the cosmic rays by detecting them. However, in the high energy interactions produced at the source, electrically neutral particles such as photons, neutrons, and neutrinos are also produced and their trajectories are not deviated, being directed from their point of origin to the observer (e.g. [23, 71, 135]). Owing to their short lifetime neutrons cannot survive the path length to the Earth (decay length $\sim 9 \mathrm{pc}$ at $1 \mathrm{PeV}$ ) and neutrinos do not interact efficiently in the atmosphere.

It is in this context that Gamma Ray Astronomy has demonstrated itself to be a powerful tool. The observations made to date have detected $\gamma$-rays from many astronomical objects, e.g. neutron stars, interstellar clouds, the center of our Galaxy and the nuclei of active galaxies (AGNs). One might expect very important implications for high energy astrophysics from the observations at energies greater than $10^{11} \mathrm{eV}$ of extragalactic sources (e.g. [105]). The fluxes of $\gamma$-rays at these energies are attenuated because of their interactions with the cosmic radio, microwave, infrared and optical radiation fields (e.g. 8, 151]). Measurements of the flux attenuation can then provide important information on the distribution of these fields. For example, the threshold energy for pair production in reactions of photons with the $2.7 \mathrm{~K}$ background radiation is reached at $10^{14} \mathrm{eV}$ and the absorption length is of the order of $\sim 7 \mathrm{kpc}$. For the infrared background, maximum absorption is reached at energies greater than $10^{12} \mathrm{eV}$.

The monograph entitled "Origin of Cosmic Rays" [78] strongly influenced subsequent papers, mainly because of the suggestive title. More than
1000 papers discussing the origin of CRs have appeared since then. Recently, Drury [58] presented a critical discussion, because we are still searching for the origin of CRs. The discussion involves the place of their origin (galactic or extragalactic), the origin of the accelerated particle, the origin of the energy, the acceleration site and mechanisms, the physical limits on the accelerator, possible synthesis, and observational tests.

\subsection{LHC RESULTS}

In the past, it was impossible to accelerate particles in Earth-bound laboratories to energies of the order of $\mathrm{TeV}$.

Now, the Large Hadron Collider (LHC) - described by Straessner et al. 163 - is able to reach TeV energies for $\mathrm{p}-\mathrm{p}$ interactions at $7 \mathrm{TeV}$ to search the Higgs boson with ATLAS [76] and CMS [171] detectors. These detectors provided the ranges of exclusion for the Higgs boson mass, and together with the data coming from other experiments, e.g. Tevatron (Craig Group talk, 2012), it has been possible to reduce the possible mass of the Higgs boson in the range $115 \div 131 \mathrm{GeV}$ (95\% C.L.). With this result, and a first hint in the mass window $124 \mathrm{GeV}<m_{\mathrm{h}}<126 \mathrm{GeV}$, Elias-Miró et al. 60] discussed a detailed investigation of the stability of the Standard Model (SM) vacuum under the hypothesis $124 \mathrm{GeV}<m_{\mathrm{h}}<126 \mathrm{GeV}$, assuming the validity of SM up to very high energy scales. For a Higgs mass in the range $124 \div 126 \mathrm{GeV}$, and for the current central values of the top mass and strong coupling constant, the Higgs potential develops an instability around $10^{11} \mathrm{GeV}$, with a lifetime much longer than the age of the Universe. However, taking into account theoretical and experimental errors, stability up to the Planck scale cannot be excluded. Stability at finite temperature implies an upper bound on the reheat temperature after inflation, which depends critically on the precise values of the Higgs and top masses. A Higgs mass in the range $124 \div 126 \mathrm{GeV}$ is compatible with very high values of the reheating temperature, without conflict with baryogenesis mechanism such as leptogenesis. Elias-Miró et al. 60 derived an upper bound on the mass of heavy right-handed neutrinos by requiring that their Yukawa couplings do not destabilize the Higgs potential.

A historic result comes from combining the results obtained with the CMS and ATLAS experiments at LHC. The results from the CMS experiment gives a mass of the observed state decaying to di-photon and four-lepton final state, with statistical significance of $5 \sigma$, of $125.3 \pm 0.6 \mathrm{GeV}$ [113]. The result from ATLAS experiment gives an excess of events at $m_{\mathrm{H}} \sim 126.5 \mathrm{GeV}$ with local significance $5.0 \sigma[77$.

Within the current uncertainties, these observations are compatible with the SM Higgs boson. However, this historic milestone marks only the beginning of a new exciting challenge for frontier physics. 


\subsection{Big Bang and Diffuse EXtRagalaCtiC BACKGROUND RADIATION}

After the Big Bang the Universe started to expand with a rapid cooling. The cosmic radiation now observed is probably a melting of various components which had their origin in various stages of the evolution as the results of different processes. This is Diffuse Extragalactic Background Radiation (DEBRA), which, if observed in different energy ranges, allows the study of many astrophysical, cosmological, and particle physics phenomena [148]. DEBRA has witnessed the whole history of the Universe, from the Big Bang to the present time.

This history is marked by three main experimental witnesses supporting the Big Bang theory (e.g. [90): the light element abundances [37; the CMBR temperature at various redshifts as determined by Srianand, Petitjean \& Ledoux [159], and the references therein; the $\mathrm{CMB}$ at $z=0$ as result of $\mathrm{COBE}\left(T_{\mathrm{CMBR}}(0)=\right.$ $2.726 \pm 0.010 \mathrm{~K}$ ), which is well fitted by a black body spectrum [125]. At $z \simeq 2.34$, the CMBR temperature is $6.0 \mathrm{~K}<T_{\mathrm{CMBR}}(2.34)<14.0 \mathrm{~K}$. The prediction from the Hot Big Bang $T_{\mathrm{CMBR}}=T_{\mathrm{CMBR}}(0)(1+z)$ gives $T_{\mathrm{CMBR}}(2.34)=9.1 \mathrm{~K}$, which is consistent with the measurement [159].

New measurements in various energy regions are improving our knowledge about DEBRA. It can be affirmed that the Big Bang theory has been proved, and in the light of recent LHC results, the standard model is definitively correct.

\subsection{Reionization of the Universe}

After the epoch of recombination (last scattering) between $\approx 3.8 \times 10^{5} \div \approx 2 \times 10^{8}$ yr $(z \approx 1000 \div 20)$, the universe experienced the so-called Dark Ages, when the dark matter halos collapsed and merged until the appearance of the first sources of light. This brought the Dark Ages to an end. The ultraviolet light from the first sources of light also changed the physical state of the gas (hydrogen and helium) that filled the Universe, from a neutral state to a nearly fully ionized state.

This was the Reionization Era when the population III stars formed and as feedback the first $\mathrm{SNe}$ and GRBs. This occurred between $\approx(2 \div 5) \times 10^{8} \mathrm{yr}$ $(z \approx 20 \div 10)$. Soon after population II stars started to form and probably the second wave of reionization occurred and stopped at $\approx 9 \times 10^{8} \mathrm{yr}(z \approx 6)$ after the Big Bang, and then the evolution of galaxies started (e.g. [56, 57]). Quasars - the brightest and most distant known objects - offer a window on the reionization era, because neutral hydrogen gas absorbs their ultraviolet light.

Reionization drastically changes the environment for galaxy formation and evolution and in a hierarchical clustering scenario, the galaxies responsible for reionization may be the seeds of the most massive galaxies in the local Universe. Reionization is the last global phase transition in the Universe. The reionization era is thus a cosmological milestone, marking the appearance of the first stars, galaxies and quasars.

There is an apparent contradiction between the WMAP5 data [59] and quasar (QSO) absorption spectra data [65. The WMAP5 data is consistent with an epoch of reionization $z_{\text {rei }} \approx 11$, while the SDSS observations suggest $z_{\text {rei }} \approx 6$. Long GRB may constitute a complementary way to study the reionization process, possibly probing $z_{\text {rei }} \approx 6$ [99, 150, 167. Moreover, an increasing number of Lyman Alpha Emitters are routinely found at $z>6$ [161].

Recent results obtained by Ouchi et al. [140] make an important contribution to the solution of this problem. Indeed, from the the Ly $\alpha$ luminosity function $(\mathrm{LF})$, clustering measurements, and Ly $\alpha$ line profiles based on the largest sample to date of 207 Ly $\alpha$ emitters at $z=6.6$ on the $1 \mathrm{deg}^{2}$ sky of Subaru/XMMNewton Deep Survey field, Ouchi et al. [140] found that the combination of various reionization models and observational results about the LF, clustering, and line profile indicates that there would exist a small decrease of the intergalactic medium's (IGM's) Ly $\alpha$ transmission owing to reionization, but that the hydrogen IGM is not highly neutral at $z=6.6$. Their neutral-hydrogen fraction constraint implies that the major reionization process took place at $z \gtrsim 7$.

The discovery of the QSOs SDSS J1148+5251 at a redshift of $6.41\left(\approx 12.6 \times 10^{9} \mathrm{yr}\right.$ ago $)$ [56], $\mathrm{J} 114816.64+525150.3$ at $z=6.43$ 66, ULAS $\mathrm{J} 1120+0641$ at $z=7.085$ (770 Myr after the Big Bang) [131] does not contradict the results found for the epoch of reionization.

The discovery of a QSO at $z>7$, together with the discovery of a GRB at $z=9.4$ [51] strongly indicate that the epoch of reionization occurred at redshift $\approx 11$, as suggested by the WMAP5 data.

However, the search for the epoch of reionization is still one of the most important open problems for understanding the formation of the first stars, galaxies and quasars. Indeed, JANUS, the proposed mission by NASA, was able to detect tens of QSOs at $z>7$, and EUCLID, an ESA mission, was probably able to detect faint QSOs at $z>8$.

\subsection{Clusters of Galaxies}

The problems of the production and transport of heavy elements seems to have been resolved. Indeed, thermally driven galactic winds, such as from M82, have shown that only active galaxies with an ongoing starburst can enrich the ICM with metals. The amounts of metals in the ICM is at least as high as the sum of the metals in all galaxies of the cluster (e.g. [173]). Several clusters of galaxies, having strong radio emission, have been associated with EGRET sources. This is an important step in clarifying the nature of many unknown EGRET sources [47]. However, no $\gamma$-ray emission from any of the monitored CGs was detected 
in the first 11 months of operation of the Fermi LAT monitoring program of CGs [6].

It is argued that cooling flows efficiently form dark matter. This has wider implications for the formation of dark matter in massive galaxies (e.g. 63, 64]).

Although many important results have coming from satellites of the last decade, the hierarchical distribution of the dark matter, and the role of the intergalactic magnetic fields in CGs are still open.

\subsection{DARK ENERGY AND DARK MATTER}

Using various methods to determine the mass of galaxies, a discrepancy has been found that suggests $\sim 95 \%$ of the universe is in a form not easily detected by our instruments and/or experiments. This form of unknown content of the universe is the sum of Dark Energy (DE) and Dark Matter (DM). For a thorough discussion about New Cosmology see [48.

The discovery of the nature of the dark energy may provide an invaluable clue for understanding the nature and the dynamics of our universe. However, there is $\sim 30 \%$ of the matter content of the universe which is dark and still requires a detailed explanation. Baryonic DM consisting of MACHOs (Massive Astrophysical Compact Halo Objects) can yield only some fraction of the total amount of Dark Matter required by CMB observations. WIMPs (Weakly Interacting Massive Particles) (non-baryonic DM) can yield the needed cosmological amount of DM and its large scale distribution provided that it is "cold" enough. Several options have been proposed so far like: i) light neutrinos with mass in the range $m_{\nu} \sim 10 \div 30 \mathrm{eV}$, ii) light exotic particles like axions with mass in the range $m_{\text {axion }} \sim 10^{-5} \div 10^{-2} \mathrm{eV}$ or weakly interacting massive particles like neutralinos with mass in the range $M_{\chi} \sim 10 \div 1000 \mathrm{GeV}$, this last option being favored at the present time (see, e.g., 61]).

EROS and MACHO, two experiments based on the gravitational microlensing, were developed. Two lines of sight have been probed intensively: the Large Magellanic Clouds (LMC) and the Small Magellanic Clouds (SMC), located $52 \mathrm{kpc}$ and $63 \mathrm{kpc}$ respectively from the Sun [142].

With 6 years of data towards the LMC, the MACHO experiment published a most probable halo fraction between 8 and $50 \%$ in the form of $0.2 M_{\odot}$ objects [11]. Most of this range is excluded by the EROS exclusion limit, and in particular the MACHO preferred value of $20 \%$ of the halo.

Among experiments for searching WIMPs dark matter candidates there is PAMELA devoted to search for dark matter annihilation, antihelium (primordial antimatter), new matter in the Universe (strangelets?), study of cosmic-ray propagation (light nuclei and isotopes), electron spectrum (local sources?), solar physics and solar modulation, and terrestrial magnetosphere. A comparison of PAMELA expectation with many other experiments has been discussed by
Morselli [130]. Bruno 34 discussed some results from PAMELA.

The search for DM is one of the main open problems in present day astroparticle physics.

\subsection{The Galactic Center}

The Galactic Center (GC) is one of the most interesting places for testing theories in which frontier physics plays a fundamental role. There is an excellent review by Mezger, Duschl \& Zylka 128, which discusses the physical state of stars and interstellar matter in the Galactic Bulge $(R \sim 0.3 \div 3 \mathrm{kpc}$ from the dynamic center of the Galaxy), in the Nuclear Bulge $(R<0.3 \mathrm{kpc})$ and in the SgrA Radio and GMC Complex (the central $\sim 50 \mathrm{pc}$ of the Milky Way). This review reports also a list of review papers and conference proceedings related to the Galactic Center, with bibliographic details. Multifrequency GC behaviour is also discussed in the review paper by Giovannelli \& Sabau-Graziati ([85] and references therein).

LaRosa et al. 122 presented a wide-field, high dynamic range, high-resolution, long-wavelength $(\lambda=$ $90 \mathrm{~cm})$ VLA image of the GC region. This is the most accurate image of the GC. It is highly obscured in optical and soft X-rays; it shows a central compact object - a black hole candidate - with $M \sim 3.6 \times$ $10^{6} M_{\odot}$ 72, which coincides with the compact radio source SgrA* [R.A. 174541.3 (hh mm ss); Dec.: -29 0022 (dd $\mathrm{mm} \mathrm{ss})]$. SgrA* in X-rays/infrared is highly variable [73].

The GC is also a good candidate for indirect dark matter observations. Indeed, Cesarini et al. 41 using the $\gamma$-ray source detected by EGRET at the GC pointed out that the spectral features of that source are compatible with the $\gamma$-ray flux induced by pair annihilations of dark matter WIMPs.

The observation of a gamma-ray line in cosmicray fluxes would be a smoking-gun signature for dark matter annihilation or decay in the Universe. Weniger 180 analyzing 43 months FERMI-LAT data in regions close to the $\mathrm{GC}$, found a $4.6 \sigma$ indication for a $\gamma$-ray line at $E \approx 130 \mathrm{GeV}$. However, the evidence for the signal is based on about 50 photons; it will take a few years of additional data to clarify its existence.

Bringmann et al. 33 and Hooper, Kelso \& Queiroz 108 analyzed and discussed the same FERMILAT data from the direction of the inner Galaxy, and derived robust yet stringent upper limits on the annihilation cross section of the dark matter, with the warning that the set of data is still poor.

After the reports of a $\gamma$-ray line feature at $\sim$ $130 \mathrm{GeV}$, Buckley \& Hooper [35], developed a model-independent approach and discussed a number of possibilities for dark matter candidates which could potentially generate a feature of this kind.

Ghez et al. 74 measured proper motions of 17 stars within 0.4 of the Galaxy's central dark mass, that reveal orbital solutions. Orbits were derived simultaneously so that they jointly constrain the central dark 
object's properties: its mass, its position, and, for the first time using orbits, its motion on the plane of the sky. The estimated central dark mass from orbital motions is $\sim 3.7 \times 10^{6}\left[R_{0} / 8 \mathrm{kpc}\right]^{3} M_{\odot}$.

Thus the study of stellar motions near the GC will provide good tests of general relativity at intermediate relativity parameters.

Despite the wide spread belief that the GC contains a black hole with a mass $M \sim 3.7 \times 10^{6} M_{\odot}$, Kundt ([121, this volume, and references therein) shows a number of maps of our GC not all of which easily found in the standard literature. All of these maps require a burning disk as their central engine, rather than a black hole.

\subsection{GAMMA-RAY BURSTS}

Theoretical description of GRBs is still an open and strongly controversial question. The fireball (FB) model [127, 146], cannon ball (CB) model [52], spinninprecessing jet (SPJ) model [67, 68], fireshell [114] model, which come directly from the electromagnetic black hole (EMBH) model (e.g. [149] and the references therein), However, each of these models conflicts with the others.

Important implications on the origin of the highest redshift GRBs are coming from the detection of the GRB 080913 at $z=6.7$ [99], GRB 090423 at $z \sim 8.2$ [168, and GRB 090429B at $z=9.4$ [51. This means that really we are approaching to the possibility of detecting GRBs at the end of Dark Era, where the first Pop III stars appeared. Izzo et al. [114] successfully discussed a theoretical interpretation of the GRB 090423 within their fireshell model.

Wang \& Dai [176] studied the high-redshift star formation rate (SFR) up to $z \simeq 8.3$ considering the Swift GRBs tracing the star formation history and the cosmic metallicity evolution in different background cosmological models including the $\Lambda \mathrm{CDM}$, quintessence, quintessence with a time-varying equation of state and brane-world models. $\Lambda$ CDMis the preferred model, but it is compared with other results.

Dust plays an important role in understanding the formation and evolution of the galaxy in the course of cosmic history. Dust absorbs and scatters UV and optical lights from young stars, making it difficult to make out how the stars were formed in galaxies using optical and NIR observations that sample the redshifted UV/optical lights of high redshift objects. Then it is important to know how the dust extinguishes the UV/optical light at high redshift, in order to understand how stars and galaxies were formed in the early universe. At high redshift, the universe was so young that core-collapse $\mathrm{SNe}$ are suspected to be the dominant source of dust production. A crucial help in understanding dust production at high redshifts was coming from the analysis of the afterglow of the GRB 071025 placed at $z \sim 5$ [115, 145. Its red color and the inflexed shape of the afterglow spectral energy distribution (SED) suggest dust extinction dominated by SNe-dust. In order to determine which kind of SNe can produce such dust, Jang et al. 115 using their independent optical/near-infrared data of GRB 071025 at a different epochs - tested SNe-dust models with different progenitor masses and dust destruction efficiencies to constrain the dust formation mechanisms. By searching for the best-fit model of the afterglow SED, Jang et al. [115] confirmed the previous claim that the dust in GRB 071025 is most likely originated from SNe. They also found that the SNe-dust model of 13 or $25 M_{\odot}$ without dust destruction fits the extinction property of GRB 071025 best, while pair-instability SNe models with a $170 M_{\odot}$ progenitor poorly fit the data.

Then, Jang et al. [115] results indicate that, at least in some systems at high redshift, SNe with intermediate masses within $10 \div 30 M_{\odot}$ were the main contributors for the dust enrichment, and the dust destruction effect due to reverse shock was negligible.

Although great progress has been made in recent years, GRB theory needs further investigation in light of the experimental data coming from old and new satellites, often coordinated, such as BeppoSAX or BATSE/RXTE or ASM/RXTE or IPN or HETE or INTEGRAL or SWIFT or AGILE or FERMI or MAXI.

\subsection{Extragalactic Background Light}

Space is filled with diffuse extragalactic background light (EBL) which is the sum of starlight emitted by galaxies through the history of the universe. High energy $\gamma$-rays traversing cosmological distances are expected to be absorbed through their interactions with the EBL by: $\gamma_{\mathrm{VHE}}+\gamma_{\mathrm{EBL}} \longrightarrow \mathrm{e}^{+} \mathrm{e}^{-}$. Then the $\gamma$-ray flux $\Phi$ is suppressed while travelling from the emission point to the detection point, as $\Phi=$ $\Phi_{0} \mathrm{e}^{-\tau(E, z)}$, where $\tau(E, z)$ is the opacity. The e-fold reduction $[\tau(E, z)=1]$ is the Gamma Ray Horizon (e.g. [29, 124]).

Direct measurement of the EBL is difficult at optical to infrared wavelengths because of the strong foreground radiation originating in the solar system. However, the measurement of the EBL is important for VHE gamma-ray astronomy, as well as for astronomers modelling star formation and galaxy evolution. Second only in intensity to the cosmic microwave background (CMB), the optical and infrared (IR) EBL contains the imprint of galaxy evolution since the Big Bang. This includes the light produced during formation and reprocessing of stars. Current measurements of the EBL are reported in the paper by Schroedter ([152] and references therein). He used the available VHE spectra from six blazars. Later, the redshift region over which the gamma reaction history can be constrained by observations has been extended up to $z=0.536$. Upper EBL limit based on 3C 279 data have been obtained [10. The universe is more transparent to VHE gamma rays than expected. Thus many more AGNs could be seen at these energies. 
Indeed, Abdo et al. 1 observed a number of TeVselected AGNs during the first 5.5 months of observations with the Large Area Telescope (LAT) on-board the Fermi Gamma-ray Space Telescope. Redshiftdependent evolution is revealed in the spectra of objects detected at $\mathrm{GeV}$ and $\mathrm{TeV}$ energies. The most reasonable explanation for this is the absorption on the EBL, and as such, it would represent the first model-independent evidence for absorption of $\gamma$-rays on the EBL. Abdo et al. [5] by using a sample of $\gamma$-ray blazars with redshift up to $z \sim 3$, and GRBs with redshift up to $z \sim 4.3$, measured by Fermi/LAT placed upper limits on the $\gamma$-ray opacity of the universe at various energies and redshifts and compared this with predictions from well-known EBL models. They found an EBL intensity in the optical-ultraviolet wavelengths that rules out the "baseline" model of Stecker, Malkan \& Scully [162] with high confidence.

\subsection{RelativistiC JETS}

Rotating massive cosmic sources can produce jets. Indeed, relativistic jets have been found in numerous galactic and extragalactic cosmic sources at different energy bands. The emitted spectra of jets from cosmic sources of different nature are strongly dependent on the angle formed by the beam axis and the line of sight, and obviously by the Lorentz factor of the particles (e.g. 24] and the references therein, [15-18, 18 22]). Thus observations of jet sources at different frequencies can provide new inputs for the comprehension of such extremely efficient carriers of energy, as for cosmological GRBs. The discovered analogy among p-QSOs, QSOs, and GRBs is fundamental for studying the common physics governing these different classes of objects via $\mathrm{\mu}$-QSOs, which are galactic, and then apparently brighter and with all processes occurring in time scales accessible by our experiments (e.g. [42]). Chaty 43 noted the importance of multifrequency observations of jet sources by means of the measurements of GRS $1915+105$.

Dermer et al. [55] suggested that ultra-high energy cosmic rays (UHECRs) could come from black hole jets of radio galaxies. Spectral signatures associated with UHECR hadron acceleration in studies of radio galaxies and blazars with FERMI observatory and ground-based $\gamma$-ray observatories can provide evidence for cosmic-ray particle acceleration in black hole plasma jets. Also in this case, $\gamma$-ray multifrequency observations $(\mathrm{MeV}-\mathrm{GeV}-\mathrm{TeV})$ together with observations of $\mathrm{PeV}$ neutrinos could confirm whether blackhole jets in radio galaxies accelerate the UHECRs.

Despite their frequent outburst activity, microquasars have never been unambiguously detected emitting high-energy gamma rays. The Fermi/LAT has detected a variable high-energy source coinciding with the position of the X-ray binary and microquasar Cygnus $\mathrm{X}-3$. Its identification with Cygnus $\mathrm{X}-3$ is secured by the detection of its orbital period in gamma rays, as well as the correlation of the LAT flux with radio emission from the relativistic jets of Cygnus $\mathrm{X}-3$. The $\gamma$-ray emission probably originates from within the binary system 2. Microquasar LS 5039 has been unambiguously detected by Fermi/LAT through its 3.9-day modulated emission. Analyzing the spectrum, variable with the orbital phase, and having a cutoff, Abdo et al. 3 concluded that the $\gamma$-ray emission of LS 5039 is magnetospheric in origin, like that of pulsars detected by Fermi. This experimental evidence of emission in the $\mathrm{GeV}$ region of microquasars opens an interesting window onto the formation of relativistic jets.

\subsection{Cataclysmic Variables}

The detection of CVs with the INTEGRAL observatory [14 has recently renewed the interest of high energy astrophysicists in such systems, and subsequently involved the low-energy astrophysical community again. The detection of $\mathrm{CVs}$ having orbital periods inside the so-called Period Gap between 2 and 3 hours, which separates polars (experiencing gravitational radiation) from intermediate polars (experiencing magnetic braking), renders attractive the idea of physical continuity between the two classes. Further investigations of this important problem are necessary. For recent reviews on CVs see the papers by Giovannelli [80] and Giovannelli \& Sabau-Graziati [95].

Type Ia supernovae (SNe Ia) are essential astrophysical tools for the study and exploration of fundamental properties in the cosmos. The first suggestion about the possibility that progenitors of SNe Ia in late-type galaxies were CVs systems was given by Della Valle \& Livio [54. Since then, the identification of the progenitors of SNe Ia remained controversial. It is now generally accepted that SNe Ia originate from binary star systems in which at least one component is a carbon-oxygen white dwarf (WD). These systems belong to the general class of CVs. Current theories for SNe Ia progenitors hold that, either via Roche lobe overflow of the companion or via a wind, the WD accumulates hydrogen or helium rich material which is then burned to $\mathrm{C}$ and $\mathrm{O}$ onto the WD's surface. However, the specifics of this scenario are far from being understood. Within this framework Kafka [117 discussed the latest attempts to identify and study those controversial SNe Ia progenitors. She also introduced the most promising progenitors in hand and presented observational diagnostic that can reveal more members of the category.

Taani et al. [165] discussed the origin of the progenitors of millisecond pulsars (MSPs) and concluded that some fraction of isolated MSPs originate from the conversion of WDs to MSPs via accretion induced collapse (AIC) process. Taani et al. [166] discussed mainly on the massive binary WDs $\left(M \geq 1.0 M_{\odot}\right)$ forming $\mathrm{CVs}$, that could potentially evolve to reach Chandrasekhar limit, thereafter they collapse and become MSPs.

$\mathrm{SN} \mathrm{Ia-CVs} \mathrm{connection} \mathrm{is} \mathrm{one} \mathrm{of} \mathrm{the} \mathrm{hottest} \mathrm{problem}$ of today astrophysics (e.g. 96]). 


\subsection{High Mass X-RAY Binaries}

For general reviews see e.g. Giovannelli \& SabauGraziati [84, 85] and van den Heuvel [104] and references therein.

HMXBs are young systems, with age $\leq 10^{7} \mathrm{yr}$, mainly located in the galactic plane (e.g. [100). A compact object - the secondary star -, mostly a magnetized neutron star (X-ray pulsar) is orbiting around an early type star $(\mathrm{O}, \mathrm{B}, \mathrm{Be})$ - the primary with $M \geq 10 M_{\odot}$. The optical luminosity of the system is dominated by the early type star.

Such systems are the best laboratory for the study of accreting processes thanks to their relative high luminosity in a large part of the electromagnetic spectrum. Because of the strong interactions between the optical companion and collapsed object, low and high energy processes are strictly related.

In X-ray/Be binaries the mass loss processes are due to the rapid rotation of the Be star, the stellar wind and, sporadically, to the expulsion of casual quantity of matter essentially triggered by gravitational effects close to the periastron passage of the neutron star. The long orbital period ( $>10$ days) and a large eccentricity of the orbit $(>0.2)$ together with transient hard X-ray behavior are the main characteristics of these systems. The whole sample of HMXBs contain 128 X-ray pulsars in the Magellanic Clouds [39] and 114 in the Milky Way [116. Only few of them have been extensively studied. Among these, the system A $0535+26 / \mathrm{HDE} 245770$ is the best known thanks to concomitant favorable causes, which rendered possible thirty seven years of coordinated multifrequency observations, most of them discussed by e.g. Giovannelli \& Sabau-Graziati [83, 90, Burger et al. [36]. Cyclotron lines have been detected in 21 X-ray pulsars ([39] and the references therein), allowing the direct determination of the magnetic field intensity at the surface of the neutron star of $\sim 10^{12} \div 10^{13} \mathrm{G}$.

Accretion powered X-ray pulsars usually capture material from the optical companion via stellar wind, since this primary star generally does not fill its Roche lobe. However, in some specific conditions (e.g. the passage at the periastron of the neutron star) and in particular systems (e.g. A $0535+26 / \mathrm{HDE} 245770$ ), it is possible the formation of a temporary accretion disk around the neutron star behind the shock front of the stellar wind. This enhances the efficiency of the process of mass transfer from the primary star onto the secondary collapsed star, as discussed by Giovannelli \& Ziolkowski 82 and by Giovannelli et al. 87] in the case of A $0535+26$.

Giovannelli \& Sabau-Graziati 93 discussed the history of the discovery of optical indicators of high energy emission in the prototype system A0535+26/HDE $245770 \equiv$ Flavia' star, updated to the March-April 2010 event when a strong optical activity occurred roughly 8 days before the X-ray outburst 38 that was predicted by Giovannelli, Gualandi \& Sabau-Graziati [91]. This optical indicator of X-ray outburst together with the whole history of A0535+26 system allowed to conclude that the periastron passage of the neutron star is scanned every 110.856 days (optical orbital period), and the anomalous and casual X-ray outbursts are triggered starting from that moment and occur roughly after roughly 8 days - the transit time for material expelled from the primary to reaching the secondary. By contrast, the normal outbursts triggered by the 'steady' stellar wind of Be star - in a state of 'quiescence' - occur at the periastron.

An alternative explanation of such delay between optical and X-ray flares could be due to the presence of a non-stationary accretion disk around the NS related to the motion of a high-mass flux region from the outer boundary of the NS Roche lobe to the Alfven surface due to action of the $\alpha$-viscosity, as suggested by Bisnovatyi-Kogan, Giovannelli \& Klepnev [30, who constructed a quantitative model of such event. For bright outbursts the 8 days delay happened at $\alpha=0.1$.

However, it is still an important, open and controversial problem in astrophysics how X-ray outbursts are triggered in X-ray pulsars.

Important data is also coming in from $\mathrm{GeV}$ observations of HMXBs. Indeed, Abdo et al. 44 present the first results from the observations of LSI $+61^{\circ} 303$ using Fermi/LAT data obtained between 2008 August and 2009 March. Their results indicate variability that is consistent with the binary period, with the emission being modulated at 26.6 days. This constitutes the first detection of orbital periodicity in high-energy $\gamma$-rays $(20 \mathrm{MeV} \div 100 \mathrm{GeV})$. The light curve is characterized by a broad peak after periastron, as well as a smaller peak just before apastron. The spectrum is best represented by a power law with an exponential cutoff, yielding an overall flux above $100 \mathrm{MeV}$ of $\simeq 0.82 \times 10^{-6} \mathrm{ph} \mathrm{cm}^{-2} \mathrm{~s}^{-1}$, with a cutoff at $\sim 6.3 \mathrm{GeV}$ and photon index $\gamma \sim 2.21$. There is no significant spectral change with orbital phase. The phase of maximum emission, close to periastron, hints at inverse Compton scattering as the main radiation mechanism. However, previous very high-energy gamma ray $(>100 \mathrm{GeV}$ ) observations by MAGIC and VERITAS show peak emission close to apastron. This and the energy cutoff seen with Fermi suggest that the link between HE and VHE gamma rays is nontrivial. This is an open problem for future investigation.

\subsubsection{Obscured Sources and Supergiant FAST X-RAY TRANSIENTS}

One of the most important contributions of the INTEGRAL satellite has been its scans of the Galactic plane and bulge, which have led to the discovery of a number of previously unknown transient X-ray binary sources (e.g. [31]). Many of these sources have high absorbing column density $\left(N_{\mathrm{H}} \approx 10^{23}\right)$, making the INTEGRAL hard X-ray response crucial for their discovery. These sources are in the course of being unveiled by means of multi-wavelength optical, near- 
and mid-infrared observations.

Other sources discovered by the INTEGRAL are the Supergiant high-mass X-ray binaries (SGXBs) (e.g. 45]). They are believed to be rare objects, as stars in the supergiant phase have a very short lifetime and to date only about two dozen of them have been discovered. They are known to be persistent and bright X-ray sources. INTEGRAL changed this classical picture, as its observations revealed the presence of a new subclass of SGXBs that have been labeled supergiant fast X-ray transients (SFXTs), since they are strongly characterized by fast X-ray outbursts lasting less than a day, typically a few hours [153, and extreme X-ray luminosity dynamic ranges $\left(10^{3 \div 5}\right)[156$. SFXTs are one of the most intriguing (and unexpected) results of the INTEGRAL mission. They are a new class of High Mass X-ray Binaries. They are composed by a massive OB supergiant star as companion donor and a compact object. At least four SFXTs host a neutron star, because X-ray pulsations have been discovered, while for the others a black hole cannot be excluded [156]. In a recent review Sidoli [155] discussed on the latest progress on SFXTs and future direction.

The importance of the discovery of this new population is based on the constraints on the formation and evolution of HMXBs 43, 44, 46, 147]: Does the dominant population of short-living systems - born with two very massive components - occur in rich star-forming region? What will happen when the supergiant star dies? Are primary progenitors of NS/NS or NS/BH mergers good candidates for gravitational wave emitters? Can we find a link with short/hard $\gamma$-ray bursts?

\subsection{ULTRA-COMPACT DOUBle-DEGENERATED BINARIES}

Ultra-compact double-degenerated binaries (UCD) consist of two compact stars, which can be black holes, neutron stars or white dwarfs. In the case of two white dwarfs revolving around each other with an orbital period $P_{\text {orb }} \leq 20$ min the separation is very small. The separation of the two components for a UCD with $P_{\text {orb }} \approx 10$ min or shorter is smaller than the diameter of Jupiter.

Smak 157] was the first to suggest that AM CVn was such a system, and Paczyński 141 discussed the possibility of gravitational waves emission from it. Later many papers appeared about UCD systems (e.g. [50, 136, 164, 174, 175, 177, 178]).

These UCD are evolutionary remnants of low-mass binaries, and they are numerous in the Milky Way. The discovery of UCD forebodes interesting hints for possible detection of gravitational waves with the LISA observatory.

Moreover, UCD are extremely important for solving the problem of the SNe Ia progenitors. Indeed, following Giovannelli \& Sabau-Graziati 95, it is well accepted by the community that Type Ia SNe are the result of the explosion of a carbon-oxygen WD that grows to near Chandrasekhar's limit $\left(M_{\mathrm{ch}} \approx 1.4 M_{\odot}\right)$ in a close binary system [109. But the debate is focussed around the different kinds of progenitors. Indeed, in the past, two families of progenitor models have been proposed. They differ in the mode of WD mass increase. The first family is the so-called single degenerate (SD) model [181, in which the WD accretes and burns hydrogen-rich material from the companion. The second family is the so-called double degenerate (DD) model, in which the merging of two WDs in a close binary triggers the explosion [112, 179]. The two scenarios produce different delay times for the birth of the binary system to explosion. Thus it is hopefully possible to discover the progenitors of Type Ia SNe by studying their delay time distribution (DDT). The DDT can be determined empirically from the lag between the cosmic star formation rate and Type Ia SN birthrate (e.g. 137]).

The DD scenario, in which two CO WDs can produce a SN Ia while merging if their combined mass is larger than $M_{\mathrm{ch}}$ has been recently discussed by Toonen, Nelemans \& Portegies Zwart [172].

\subsection{Magnetars}

The discovery of magnetars (Anomalous X-ray Pulsars - AXPs - and Soft Gamma-ray Repeaters SGRs) is another very exciting result in recent years ([126, 143 and e.g. review by Giovannelli \& SabauGraziati 85 ] and the references therein). Indeed, with the magnetic field intensity of order $10^{14} \div 10^{15} \mathrm{G}$ a question naturally arises: what kind of SN produces such AXPs and SGRs? Are really the collapsed objects in AXPs and SGRs neutron stars? (e.g. [111, 144]). With such high magnetic field intensity an almost 'obvious' consequence can be derived: the correspondent dimension of the source must be of $\sim 10 \mathrm{~m}$ [86]. This could be the dimension of the acceleration zone in supercompact stars. Could they be quark stars?

Ghosh [75] discussed some of the developments in quark star physics, along with the consequences of possible hadron-to-quark phase transition, in a highdensity scenario of neutron stars, and their implications for Astroparticle Physics.

Important consequences could be derived by experimentally demonstrated the continuity among rotationpowered pulsars, magnetars, and millisecond pulsars [110, 119]. However, the physical reason for this continuity is not yet clear.

Several papers have appeared about the possibility of having quark stars (e.g. [97, 138]). Strange quark matter could be found in the core of neutron stars or forming strange quark stars. Quarks and electrons interact with the magnetic field via their electric charges and anomalous magnetic moments. In contrast to the magnetic field value of $10^{19} \mathrm{G}$, obtained when anomalous magnetic moments are not taken into account, González Felipe et al. 97 found the upper bound $B \lesssim 8.6 \times 10^{17} \mathrm{G}$, for the stability 
of the system. A phase transition could be hidden for fields greater than this value.

An analytical model of a magnetar as a highdensity magnetized quark bag was discussed by Orsaria, Ranea-Sandoval \& Vucetich [138].

They considered the effect of strong magnetic fields $\left(B>5 \times 10^{16} \mathrm{G}\right)$ in the equation of state. They found an analytic expression for the mass-radius (M-R) relationship from the energy variational principle in general relativity, and the results were compared with observational evidence of possible quark and/or hybrid stars.

The M- $\mathrm{R}$ relationship, gravitational redshift and rotational Kepler periods of a magnetized quark-hybrid stars were compared with those of standard neutron stars [139.

\section{Cross Sections of Nuclear Reactions in Stars}

Knowledge of the cross-sections of nuclear reactions occurring in the stars is one of the most crucial points in all astroparticle physics. Direct measurements of the cross sections of the ${ }^{3} \mathrm{He}\left({ }^{4} \mathrm{He}, \gamma\right){ }^{7} \mathrm{Be}$ and ${ }^{7} \mathrm{Be}(\mathrm{p}, \gamma){ }^{8} \mathrm{Be}$ reactions of the $\mathrm{p}-\mathrm{p}$ chain and ${ }^{14} \mathrm{~N}(\mathrm{p}, \gamma){ }^{15} \mathrm{O}$ reaction of the CNO-cycle will allow a substantial improvement in our knowledge on stellar evolution.

The LUNA collaboration has already measured the key reactions $\mathrm{D}(\mathrm{p}, \gamma){ }^{3} \mathrm{He},{ }^{3} \mathrm{He}(\mathrm{D}, \mathrm{p}){ }^{4} \mathrm{He}$ and ${ }^{3} \mathrm{He}\left({ }^{4} \mathrm{He}, \gamma\right){ }^{7} \mathrm{Be}$ with good accuracy. These measurements substantially reduces the theoretical uncertainty of $\mathrm{D},{ }^{3} \mathrm{He},{ }^{7} \mathrm{Li}$ abundances. The $\mathrm{D}\left({ }^{4} \mathrm{He}, \gamma\right){ }^{6} \mathrm{Li}$ cross section, which is the key reaction for the determination of the primordial abundance of ${ }^{6} \mathrm{Li}$, will be measured in the near future [101, 102].

There is a mission (DUAL) for nuclear astrophysics studies, which naturally addresses the requirement for medium-sensitivity large-scale exposures, and very deep pointed observations [12].

DUAL will study the origin and evolution of the elements and explores new frontiers of physics: extreme energies that drive powerful stellar explosions and accelerate particles to macroscopic energies; extreme densities that modify the laws of physics around the most compact objects known; and extreme fields that influence matter in a way that is unknown on Earth.

The roadmap of high energy astronomy shows a white spot on the future track of nuclear astrophysics i.e. in the energy range of $100 \mathrm{keV} \div 100 \mathrm{MeV}$, which will be explored by the DUAL mission [13].

\section{Neutrino Astronomy}

For a short discussion about neutrino astronomy see e.g. the paper by Giovannelli ([87] and the references therein), as well as all the papers of the Neutrino Astronomy Session, which appeared in the proceedings of the Vulcano Workshops 2006, 2008, 2010, and 2012 [88, 89]. However, it is important to note that several papers appeared about: i) the sources of $\mathrm{HE}$ neutrinos 9 and diffuse neutrinos in the Galaxy 62; ii) potential neutrino signals from galactic $\gamma$-ray sources [118]; iii) galactic cosmic-ray pevatrons with multi-TeV $\gamma$-rays and neutrinos [70; iv) results achieved with AMANDA: 32 galactic and extragalactic sources have been detected [182; diffuse neutrino flux from the inner Galaxy 169]; discussion about VHE neutrino astronomic experiments [40]. Important news and references can be found in the proceedings of the Les Rencontres de Physique de la Vallée d'Aoste [98].

News about the neutrino oscillations have been reported by Mezzetto 129. The angle $\Theta_{13}$ differs from zero: $\sin ^{2} \Theta_{13}=0.013$. This result opens the door to $\mathrm{CP}$ violation searches, with profound implications for our understanding of the matter-antimatter asymmetry in the universe. This result is extremely important in view of our understanding of the physics governing the Universe. Indeed, it confirms the predictions of Boyarsky, Ruchayskiy \& Shaposhnikov 32 at the end of their comprehensive overview of an extension of the Standard Model that contains three right-handed (sterile) neutrinos with masses below the electroweak scale [the Neutrino Minimal Standard Model, ( $\mathrm{MMSM})]$. They considered the history of the Universe from the inflationary era until the present day, and demonstrated that most of the observed phenomena beyond the Standard Model can be explained within the framework of this model. They reviewed the mechanism of baryon asymmetry of the Universe in the $\vee$ MSM and discussed a dark matter candidate that can be warm or cold and satisfies all existing constraints. From the viewpoint of particle physics the model provides an explanation for neutrino flavor oscillations.

\section{Conclusions And Reflections}

This review is far from complete, but I will now conclude with some comments about the topics discussed here.

(1.) Many ground-based and space-based experiments are exploring the whole energy range of the CR spectrum from $\sim 1 \mathrm{GeV}$ to $\sim 10^{12} \mathrm{GeV}$ and many experiments are programmed for the near future. A significant improvement has been made in the definition of the CR spectrum for protons, electrons, positrons, antiprotons, and all ions. Better results are expected in the near future. Particular interest is devoted to the knowledge of the extreme $\mathrm{HE}$ energy CRs.

(2.) Many experiments are exploring cosmic sources along the whole electromagnetic spectrum, and new space-based and ground-based experiments are developing a tendency to explore processes at higher and higher energies, which are directly linking photonic astrophysics with particle astrophysics.

(3.) A particular attention is necessary at the highest energies where the cosmic ray spectrum extends 
to $10^{20} \mathrm{eV}$ (see Fig. 1). However the origins of the spectacularly high-energy particles remains obscure. Particle energies of this magnitude imply that near their acceleration sites a range of elementary particle physics phenomena is present which is beyond the ability of present day particle accelerators to explore. VHE $\gamma$-ray astronomy may catch a glimpse of these phenomena.

It is becoming increasingly clear that the energy régime covered by $\mathrm{VHE} \gamma$-ray astronomy will be able to address a number of significant scientific questions, which include: i) What parameters determine the cutoff energy for pulsed $\gamma$-rays from pulsars? ii) What is the role of shell-type supernovae in the production of cosmic rays? iii) At what energies do AGN blazar spectra cut-off? iv) Are gamma blazar spectral cut-offs intrinsic to the source or due to intergalactic absorption? v) Is the dominant particle species in AGN jets leptonic or hadronic? vi) Can intergalactic absorption of the VHE emission of AGN's be a tool to calibrate the epoch of galaxy formation, the Hubble parameter, and the distance to $\gamma$-ray bursts? vii) Are there sources of $\gamma$-rays which are 'loud' at VHEs, but 'quiet' at other wavelengths?

The Multifrequency Astrophysics and Multienergy Particle Astrophysics observations allow us to complement one observation with others thus improving our potential deciphering of the problems and understanding.

There are many problems in performing simultaneous Multifrequency, Multienergy Multisite, Multiinstrument, Multiplatform measurements due to: i) objective technological difficulties; ii) sharing common scientific objectives; iii) problems of scheduling and budgets; iv) politic management of science.

In spite of the many ground-based and space-based experiments, which provide an impressive quantity of excellent data in various energy regions, many open problems still remain. I believe that only a drastic change in the philosophy of the experiments will enable the open problems to be solved more rapidly. For example, in the case of space-based experiments, it is necessary to support small satellites, dedicated to specific missions and problems, and providing the possibility to schedule very long-term observations, because they are faster to prepare, easier to manage and less expensive to run than medium-size and large satellites. In addition, because they can be prepared faster, it is possible to use more recent technologies in small satellites, than in bigger satellites, in which, typically, 15-year-old (or older) technologies are used.

In addition, the international community needs to be able to persuade the various national and international space agencies to give strong support for arrays of small satellites, each of them specialized in a particular energy band or in the study of a specific physical process.

I strongly believe that in the coming decades spacebased, ground-based and maybe lunar-based passive physics experiments will be the most suitable probes for sounding the physics of the Universe. Active physics experiments have probably already reached the maximum dimensions compatible with a reasonable cost/benefit ratio, with the obvious exception of neutrino-astronomy experiments.

\section{ACKNOWLEDGEMENTS}

Many thanks to Professor Giora Shaviv for helpful suggestions that have improved the quality of this paper. This research has made use of NASA's Astrophysics Data System.

\section{REFERENCES}

[1] Abdo, A.A. et al.: 2009a, ApJ 707, 1310

[2] Abdo, A.A. et al.: 2009b, Sci. 326, 1512

[3] Abdo, A.A. et al.: 2009c, ApJ 706, L56

[4] Abdo, A.A. et al.: 2009e, ApJ 701L, 123

[5] Abdo, A.A. et al.: 2010, ApJ 723, 1082

[6] Ackermann, M. et al.: 2010, ApJ 717, L71

[7] Ackermann, M. et al.: 2011, Phys. Rev. Letter 107, 241302

[8] Aharonian, F.A.: 2004, Very High Energy Cosmic Gamma Radiation: A Crucial Window on the Extreme Universe, World Scientific Publishing Co. Pte. Ltd., 99-135

[9] Aharonian, F.A.: 2007, Sci. 315, 70

[10] Albert, J. et al. (MAGIC Collaboration): 2008, Sci. 320,1752

[11] Alcock, C. et al.: 2000, ApJ 542, 281

[12] von Ballmoos, P., Takahashi, T., Boggs, S.E.: 2010 , Nucl. Instr. \& Meth. Phys. Res. 623, 431

[13] von Ballmoos, P. et al. (The DUAL Consortium): 2012, Exp. Astron. 34, 583

[14] Barlow, E.J. et al.: 2006, MNRAS 372, 224

[15] Beall, J.H.: 2002, in Multifrequency Behaviour of High Energy Cosmic Sources, F. Giovannelli \& L. Sabau-Graziati (eds.), Mem. SAIt 73, 379

[16] Beall, J.H.: 2003, ChJA\&AS 3, 373

[17] Beall, J.H.: 2008, ChJA\&AS 8, 311

[18] Beall, J.H.: 2009, in Frontier Objects in Astrophysics and Particle Physics, F. Giovannelli \& G. Mannocchi, (eds.), SIF, Ed. Compositori, Bologna, Italy, 98, 283

[19] Beall, J.H., Guillory, J., Rose, D.V.: 1999, in Multifrequency Behaviour of High Energy Cosmic Sources, F. Giovannelli \& L. Sabau-Graziati (eds.), Mem. SAIt 70, 1235

[20] Beall, J.H. et al.: 2006, ChJA\&AS1 6, 283

[21] Beall, J.H. et al.: 2007, in Frontier Objects in Astrophysics and Particle Physics, F. Giovannelli \& G. Mannocchi, (eds.), SIF, Ed. Compositori, Bologna, Italy, 93,315

[22] Beall, J.H., Guillory, J., Rose, D.V.: 2009, in Frontier Objects in Astrophysics and Particle Physics, F. Giovannelli \& G. Mannocchi, (eds.), SIF, Ed.

Compositori, Bologna, Italy, 98, 301 
[23] Beatty, J.J. et al.: 2011, CERN web page, rpp2011-rev-cosmic-rays

[24] Bednarek, W., Giovannelli, F., Karakula, S., Tkaczyk, W.: 1990, A\&A 236, 268

[25] Bennett, C.L. et al.: 2003, ApJ 583, 1

[26] Bernabei, R. et al.: 2011, in Frontier Objects in Astrophysics and Particle Physics, F. Giovannelli \& G. Mannocchi (eds.), SIF, Ed. Compositori, Bologna, Italy 103, 157

[27] Bernabei, R. et al.: 2013, this volume

[28] Biermann, P. L.: 1999, Astrophys. \& Space Sci. 264, 423

[29] Blanch, O., Martinez, M.: 2005, Astrop. Phys. 23, 588

[30] Bisnovatyi-Kogan, G.S., Giovannelli, F., Klepnev, A.: 2012, Invited Talk presented at COSPAR 2012, E1.2-0025-12

[31] Bodaghee, A. et al.: 2007, A\&A 467, 585

[32] Boyarsky, A., Ruchayskiy, O., Shaposhnikov, M.: 2009, Ann. Rev. Nucl. Part. Sci. 59, 191

[33] Bringmann, T. et al.: 2012, J. Cosm. \& Ap. Phys., Issue 07, id. 054

[34] Bruno, A.: 2011, in Frontier Objects in Astrophysics and Particle Physics, F. Giovannelli \& G. Mannocchi (eds.), SIF, Ed. Compositori, Bologna, Italy, 103, 139

[35] Matthew R. Buckley, M.R., Hooper, D.: 2012, Phys. Rev D 86, 043524

[36] Burger, M. et al.: 1996, in Multifrequency Behaviour of High Energy Cosmic Sources, F. Giovannelli \& L. Sabau-Graziati (eds.), Mem. SAIt 67, 365

[37] Burles, S., Nollet, K.M., Turner, M.S.: 2001, ApJL $552, \mathrm{~L} 1$

[38] Caballero, I. et al.: 2010, ATEL No. 2541

[39] Caballero, I., Wilms, J.: 2012, in Multifrequency Behaviour of High Energy Cosmic Sources, F. Giovannelli \& L. Sabau-Graziati (eds.), Mem. SAIt. 83, 230

[40] Cao, Z.: 2008, Nucl. Phys. B (Proc. Suppl.) 175-176, 377

[41] Cesarini, A. et al.: 2004, Ap. Phys. 21, 267

[42] Chaty, S.: 1998, Ph.D. thesis, University Paris XI

[43] Chaty, S., 2007, in Frontier Objects in Astrophysics and Particle Physics, F. Giovannelli \& G. Mannocchi (eds.), SIF, Ed. Compositori, Bologna, Italy, 93, 329

[44] Chaty, S.: 2008, ChJA\&AS 8, 197

[45] Chaty, S.: 2010, in High Energy Phenomena in Massive Stars, J. Martí, P.L. Luque-Escamilla \& J.A. Combi (eds.), ASP Conf. Ser. 422, 243

[46] Chaty, S., Filliarte, P.: 2005, ChJA\&AS 5, 104

[47] Colafrancesco, S.: 2002, A\&A 396, 31

[48] Colafrancesco, S.: 2003, in Frontier Objects in Astrophysics and Particle Physics, F. Giovannelli \& G. Mannocchi (eds.), SIF, Ed. Compositori, Bologna, Italy, 85,141

[49] Craig, H. Group: 2012, talk presented at the APS April Meeting on 100 years of cosmic ray physics
[50] Cropper, M., Ramsay, G., Wu, K., Hakala, P.: 2004, in Magnetic Cataclysmic Variables, IAU Coll. 190, Sonja Vrielmann \& Mark Cropper (eds.), ASP Conf. Proc. 315, 324

[51] Cucchiara, A. et al.: 2011, ApJ 736, 7

[52] Dar, A., De Rújula, A.: 2004, Phys. Rep. 405, 203

[53] De Angelis, A., Mansutti, O., Persic, M.: 2008, Il N. Cim. 31 N. 4, 187

[54] Della Valle, M., Livio, M.: 1994, ApJ 423, L31

[55] Dermer, C.D., Razzaque, S., Finke, J.D., Atoyan, A.: 2009, New J. of Phys. 11, 1

[56] Djorgovski, S.G.: 2004, Nature 427, 790

[57] Djorgovski, S.G.: 2005, in The Tenth Marcel Grossmann Meeting, M. Novello, S. Perez Bergliaffa \& R. Ruffini (eds.), World Scientific Publishing Co., p. 422

[58] Drury, L.O'C.: 2012, arXiv:1203.3681v1

[59] Dunkley, J. et al.: 2009, ApJ 701, 1804

[60] Elias-Miró, J. et al.: 2012, PhLB 709, 222

[61] Ellis, J.: 2002, astro-ph 4059 (arXiv:hep-ex/0210052)

[62] Evoli, C., Grasso, D., Maccione, L.: 2007, astro-ph 0701856

[63] Fabian, A.C.: 1994, Ann. Rev. A\&A 32, 277

[64] Fabian, A.C., Nulsen, P.E.J., Canizares, C.R.: 1991, A\&A Rev. 2, 191

[65] Fan, X., Carilli, C.L., Keating, B.: 2006, ARA\&A 44, 415

[66] Fan, X. et al.: 2003, AJ 125, 1649

[67] Fargion, D.: 2003, ChJA\&AS 3, 472

[68] Fargion, D., Grossi, M.: 2006, ChJA\&AS1 6, 342

[69] di Felice, V. et al.: 2010, in 38th COSPAR Scientific Assembly, p. 5

[70] Gabici, S., Aharonian, F.A.: 2007, ApJL 665, L131

[71] Gaisser, T.K., Stanev, T.: 2000, The Eur. Phys. J. C - Particles and Fields 15 (Ns 1-4), 150

[72] Genzel, R. et al.: 2003a, ApJ 594, 812

[73] Genzel, R. et al.: 2003b, Nature 425, 934

[74] Ghez, A.M. et al.: 2005, ApJ 620, 744

[75] Ghosh, S.K.: 2009, in Frontier Objects in Astrophysics and Particle Physics, F. Giovannelli \& G. Mannocchi, (eds.), SIF, Ed. Compositori, Bologna, Italy, 98, 243

[76] Gianotti, F. (ATLAS Collaboration): 2011, talk at CERN public seminar, Dec. 13, 2011;

ATLAS-CONF-2011-163

[77] Gianotti, F. (ATLAS Collaboration): 2012, talk at CERN public seminar, July 4, 2012

[78] Ginzburg, V.L., Syrovatskij, S.I.: 1966, Sov. Phys. Uspekhi 9 (N. 2), 223

[79] Giovannelli, F.: 2007; 2009; 2011, in Frontier Objects in Astrophysics and Particle Physics, F. Giovannelli \& G. Mannocchi (eds.), SIF, Ed. Compositori, Bologna, Italy, 93, 3; 98, 3; 103, 3

[80] Giovannelli, F.: 2008, Chinese J. A\&A S 8, 237 
[81] Giovannelli, F.: 2012, in 9th IBWS 2012 Proceedings Acta Polytechnica (in press)

[82] Giovannelli, F., Ziółkowski, J.: 1990, AcA 40, 95

[83] Giovannelli, F., Sabau-Graziati, L.: 1992, Space Sci. Rev. 59, 1

[84] Giovannelli, F., Sabau-Graziati, L.: 2001, Ap\&SS 276,67

[85] Giovannelli, F., Sabau-Graziati, L.: 2004, Space Sci. Rev. 112, 1

[86] Giovannelli, F., Sabau-Graziati, L.: 2006, ChJA\&AS1 6,1

[87] Giovannelli, F., Bernabei, S., Rossi, C., Sabau-Graziati, L.: 2007, A\&A 475, 651

[88] Giovannelli, F., Mannocchi, G. (eds.): 2007; 2009; 2011, Proc. Vulcano Workshops on Frontier Objects in Astrophysics and Particle Physics, SIF, Ed. Compositori, Bologna, Italy, Vols. 93; 98; 103

[89] Giovannelli, F., Mannocchi, G. (eds.): 2013, this volume

[90] Giovannelli, F., Sabau-Graziati, L.: 2008, ChJA\&AS 8,1

[91] Giovannelli, F., Gualandi, R., Sabau-Graziati, L.: 2010, ATEL No. 2497

[92] Giovannelli, F., Sabau-Graziati, L.: 2010, in Multifrequency Behaviour of High Energy Cosmic Sources, F. Giovannelli \& L. Sabau-Graziati (eds.), Mem. SAIt 81 N. 1, 18

[93] Giovannelli, F., Sabau-Graziati, L.: 2011, Acta Polyt. 51 N. 2,21

[94] Giovannelli, F., Sabau-Graziati, L.: 2012a, in Multifrequency Behaviour of High Energy Cosmic Sources, F. Giovannelli \& L. Sabau-Graziati (eds.), Mem. SAIt 83 N. 1, 17

[95] Giovannelli, F., Sabau-Graziati, L.: 2012b, in The Golden Age of Cataclysmic Variables and Related Objects, F. Giovannelli \& L. Sabau-Graziati (eds.), Mem. SAIt 83 N. 2, (in press)

[96] Giovannelli, F., Sabau-Graziati, L. (eds.): 2012c, The Golden Age of Cataclysmic Variables and Related Objects, Mem. SAIt 83 N. 2, (in press)

[97] González Felipe, R., Pérez Martínez, A., Pérez Rojas, H., Orsaria, M.: 2008, Phys. Rev. C 77, Issue 1, 015807

[98] Greco, M. (ed.): 2009, 2010, Le Rencontres de Physique de la Vallée d'Aoste: Results and Perspectives in Particle Physics, Frascati Phys. Ser. Vol. L, Vol. LI

[99] Greiner, J. et al.: 2009, ApJ 693, 1610

[100] Grimm, H.-J.: 2003, PhD Thesis, Ludwig-Maximilians-Universität, München, Germany

[101] Gustavino, C.: 2007; 2009; 2011, in Frontier Objects in Astrophysics and Particle Physics, F. Giovannelli \& G. Mannocchi (eds.), SIF, Ed. Compositori, Bologna, Italy, 93, 191; 98, 77; 103, 657

[102] Gustavino, C.: 2013, this volume

[103] Hess, V.F.: 1912, Physik Zh. 13, 1084

[104] van den Heuvel, E.P.J.: 2009, Ap\&SS Library 359, 125
[105] Hillas, A.M., Johnson, A.P.: 1990, Proc. 21st Intern. Cosmic Ray Conf. (Adelaide) 4, 19

[106] Hooper, D., Blasi, P., Serpico, P.D.: 2009, J. Cosm. \& Ap. Phys., Issue 01, 1

[107] Hooper, D., Stebbins, A., Zurek, K.M.: 2009, Phys. Rev. D 79, 103513

[108] Hooper, D., Kelso, C., Queiroz, F.S.: 2012, arXiv:1209.3015v1

[109] Hoyle, F., Fowler, W.A.: 1960, ApJ, 132, 565

[110] Hunter, : et al: 2009, Astro2010: The Astronomy and Astrophysics Decadal Survey, Science White Papers, No. 137

[111] Hurley, K.: 2008, ChJA\&AS 8, 202

[112] Iben, I., Jr., Tutukov, A.V.: 1984, ApJS, 54, 335

[113] Incandela, J. (CMS Spokesperson): 2012, talk at CERN public seminar, July 4, 2012

[114] Izzo, L. et al.: 2010, J. Korean Phys. Soc. 57, No. 3, 551

[115] Jang, M. et al.: 2011, ApJ, 741, L20

[116] Johnstone, Wm.R.: 2005, http://www.johnstonsarchive.net/relativity/ binpulstable.html

[117] Kafka, S.: 2012, J. Astron. Space Sci. 29(2), 163 http://dx.doi.org/10.5140/JASS.2012.29.2.163

[118] Kappes, A., Hinton, J., Stegman, C., Aharonian, F.A.: 2007, ApJ 656, 870

[119] Kuiper, L.: 2007, Talk presented at the Frascati Workshop on Multifrequency Behaviour of High Energy Cosmic Sources

[120] Kulikov, G.V., Khristiansen, G.B.: 1958, JETP 35, 63

[121] Kundt, W.: 2013, this volume

[122] LaRosa, T.N., Kassim, N.E., Lazio, T.J.W., Hyman, S.D.: 2000, AJ 119, 207

[123] Lawrence, M.A., Reid, R.J.O., Watson, A.A.: 1991, J. Phys. G: Nucl. Part. Phys. 17, 733

[124] Martinez, M.: 2007, Ap\&SS 309, 477

[125] Mather, J.C. et al.: 1994, ApJ 420, 439

[126] Mereghetti, S., Stella, L.: 1995, ApJL 442, L17

[127] Meszaros, P., Rees, M.J.: 1992, ApJ 397, 570

[128] Mezger, P.G., Duschl, W.J., Zylka, R.: 1996, A\&A Rev. 7, 289

[129] Mezzetto, M.: 2011, Journal of Physics: Conference Series 335, 012005

[130] Morselli, A.: 2007, in High Energy Physics ICHEP '06, Y. Sissakian, G. Kozlov \& E. Kolganova (eds.), World Sci. Pub. Co., p. 222

[131] Mortlock, D.J. et al.: 2011, Nature 474, 616

[132] Mostafa, M.: 2012, talk presented at the APS April Meeting on 100 years of cosmic ray physics

[133] Nagano, M. et al.: 1992, J. Phys. G: Nucl. Part. Phys. 18, 423

[134] Nagano, M., Watson, A.A.: 2000, Rev. Mod. Phys. 72,689 
[135] Nakamura, K. et al. (Particle Data Group): 2010, J. Phys. G: Nucl. Part. Phys. 37, 075021

[136] Nelemans, G., Jonker, P.G.: 2010, New Astron. Rev. 54,87

[137] Nelemans G., Toonen, S., Bours, M.: 2012, arXiv:1204.2960v2

[138] Orsaria, M., Ranea-Sandoval, I.F., Vucetich, H.: 2011, ApJ 734, 410

[139] Orsaria, M., Ranea-Sandoval, I.F., Vucetich, H., Weber, F.: 2011, Int. J. Mod. Phys. E 20, 25

[140] Ouchi, M. et al.: 2010, ApJ 723, 869

[141] Paczyński, B.: 1967, AcA 17, 277

[142] Palanque-Delabrouille, N.: 2003, in Frontier Objects in Astrophysics and Particle Physics, F. Giovannelli \& G. Mannocchi (eds.), SIF, Ed. Compositori, Bologna, Italy, 85, 131

[143] van Paradijs, J., Taam, R.E., van den Heuvel, E.P.J.: 1995, A\&A 299, L41

[144] Pérez Martínez, A., Pérez Rojas, H., Mosquera Cuesta, H.J.: 2003, The European Phys. J. C 29, Issue 1,111

[145] Perley, D.A. et al.: 2010, MNRAS 406, 2473

[146] Piran, T.: 1999, Phys. Rep. 314, 575

[147] Rahoui, F., Chaty, S., Lagage, P.-O, Pantin, E.: 2008, A\&A 484, 801

[148] Ressel, M.T., Turner, M.S.: 1990, Comm. Astrophys. 14, 323

[149] Ruffini, R. et al.: 2003, AIP Conf. Proc. 668, 16

[150] Salvaterra, R. et al.: 2009, Mem, SAIt. 80, 26

[151] Schlickeiser, R.: 2003, Cosmic Ray Astrophysics, Springer-Verlag Berlin Heidelberg New York

[152] Schroedter, M.: 2005, ApJ 628, 617

[153] Sguera, V. et al.: 2006, ApJ 646, 452

[154] Shaviv, N.J., Nakar, E., Piran, T.: 2009, PhRvL 103, 111302 .

[155] Sidoli, L.: 2011, Adv. Space Res. 48, 88

[156] Sidoli, L. et al.: 2010, arXiv:1001.3234v1

[157] Smak, J.: 1967, AcA 17, 255

[158] Spergel, D.N. et al.: 2003, ApJS 148, 175

[159] Srianand, R., Petitjean, P., Ledoux, C.: 2000, Nature 408, 931

[160] Stanev, T.: 2010, High Energy Cosmic Rays, Springer Praxis Books, Springer-Verlag Berlin Heidelberg
[161] Stark, D.P., Loeb, A., Ellis, R.S.: 2007, ApJ 668, 627

[162] Stecker, F.W., Malkan, M.A., Scully, S.T.: 2006, ApJ 648, 774

[163] Straessner, A. (on behalf of the ATLAS Collaboration): 2011, in Frontier Objects in Astrophysics and Particle Physics, F. Giovannelli \& G. Mannocchi (eds.), SIF, Ed. Compositori, Bologna, Italy, 103, 43

[164] Strohmayer, T.E.: 2005, ApJ 627, 920

[165] Taani, A. et al.: 2012a, Astron. Nachr/AN 333, No. 1,53

[166] Taani, A. et al.: 2012b, arXiv:1201.3779v2

[167] Tagliaferri, G. et al.: 2005, A\&A 443, 1

[168] Tanvir, N.R. et al.: 2009, Nature 461, 1254

[169] Taylor, A.M. et al.: 2008, in High Energy Gamma-Ray Astronomy, AIP Conf. Proc. 1085, 384

[170] Thompson, D.J.: 2008, Rep. Progr. Phys. 71, Issue 11, pp. 116901

[171] Tonelli, G. (CMS Collaboration): 2011, talk at CERN public seminar, Dec. 13, 2011

[172] Toonen, S., Nelemans, G., Portegies Zwart, S.: 2012, arXiv1208.6446

[173] Tozzi, P. et al.: 2003, ApJ 593, 705

[174] Tutukov, A., Yungelson, L.Y.: 1996, MNRAS 280, 1035

[175] Ulla, A.: 1994, Space Sci. Rev. 67, 241

[176] Wang, F.Y., Dai, Z.G.: 2009, MNRAS 400, 10

[177] Warner, B.: 1995, Ap\&SS 225, 249

[178] Warner, B., Robinson, E.L.: 1972, MNRAS 159, 101

[179] Webbink, R.F.: 1984, ApJ, 277, 355

[180] Weniger, C.: 2012, J. Cosm. \& Ap. Phys. Issue 08, article id. 007

[181] Whelan, J., Iben, I. Jr.: 1973, ApJ, 186, 1007

[182] Xu, X.W. (IceCube Collaboration): 2008, N. Phys. B $175-176,401$

[183] Zatsepin, V.I.: 1995, J. Phys. G: Nucl. Part. Phys. 21, Issue 5, L31 